FFP12 2011

Udine 21-23 November 2011

\title{
RESULTS FROM THE ATLAS EXPERIMENT AT THE LHC
}

Antonio Sidoti, Istituto Nazionale Fisica Nucleare - Sezione Roma, Rome, Italy on behalf of the ATLAS collaboration

\begin{abstract}
ATLAS is a general purpose detector located at one of the four interaction points of the Large Hadron Collider at the CERN laboratory near Geneva, Switzerland. In 2010 and 2011, LHC collided proton beams at the unprecedented center of mass energy of $7 \mathrm{TeV}$ and the ATLAS detector has collected more than $5.25 \mathrm{fb}-$ ${ }^{1}$ of data. The detector performance and results for Standard Model and Beyond the Standard Model physics are presented here.
\end{abstract}

\section{Introduction}

The LHC collider at CERN started its operation with first proton-proton collisions in 2009. After a commissioning phase, thanks to the excellent performance of the CERN Accelerator Division crew, the ATLAS detector collected $5.25 \mathrm{fb}^{-1}$ of proton-proton collisions at $\sqrt{s}=7 \mathrm{TeV}$ respectively in 2011. In these proceedings, we will report on the latest physics results obtained by the ATLAS experiment. We will show the Standard Model (SM) physics measurements performed with already competitive precision with respect to former collider experiment. Comparing those against predictions from SM will lead to evidences of Physics Beyond Standard Model if discrepancies are found. Direct searches of SM Higgs boson, supersymmetric or exotic physics searches will be shown.
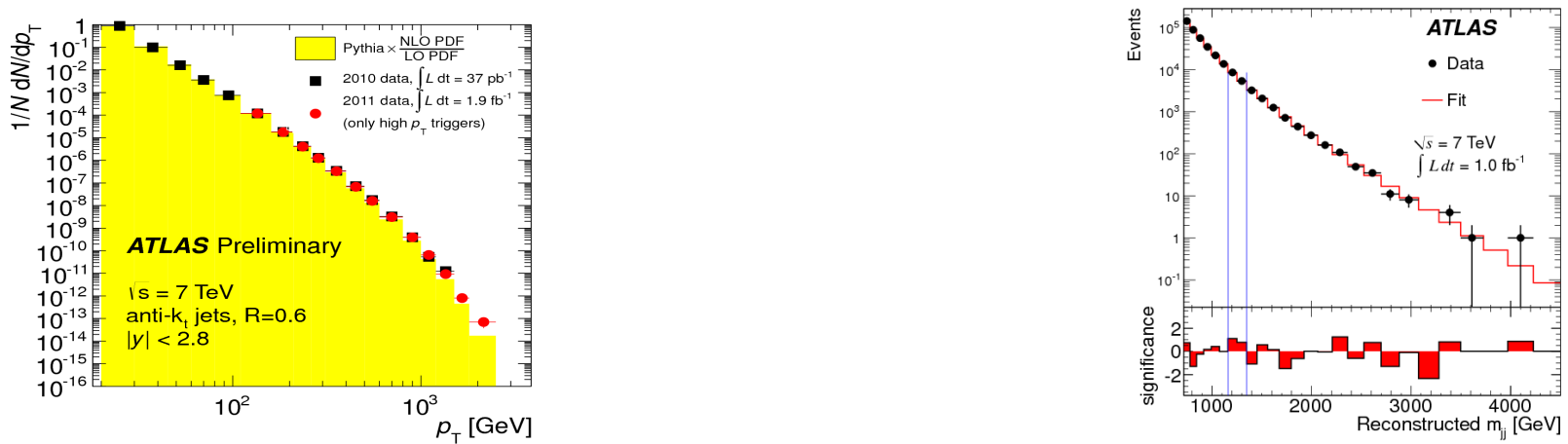

Figure 1: Left: Inclusive Jet $P_{T}$ differential cross section do/dP $P_{T}$ measured with 2010 and 2011 data (ATLAS 2011a). Right: Dijet mass distribution. The blue lines indicates the mass region where the deviation from the fit with a smooth functional form describing the QCD background theoretical expectations is more important. However, the significance of this region is well below the 2 standard deviations (ATLAS 2011b).

\section{Standard Model Physics Measurements}

A detailed description of the ATLAS detector can be found in (Aad 2008). The more important SM processes have been tested at the unprecedented center of mass energy of $7 \mathrm{TeV}$. We will show various SM measurements performed by ATLAS starting from processes with larger cross sections to the ones with smaller ones.

\subsection{Hard QCD Results}

The inclusive jet and the dijet mass cross section are measured in different rapidity regions. The inclusive jet measurement is performed in the $p_{\mathrm{T}}$ range $20 \mathrm{GeV}-1.5 \mathrm{TeV}^{1}$, and it extends to a wide rapidity range $(|\mathrm{y}|<4.4)$ (Figure 1: Left). Jets are reconstructed using the anti- $\mathrm{k}_{\mathrm{T}}$ algorithm with different $R$ distance parameters $(R=0.4$ and $R=0.6)$. These different $R$ parameters make the jets differently sensitive to the hadronization, underlying event and pile up. Jet energies are corrected for detector effects taking into account non linearity due to the different calorimetric response to

\footnotetext{
${ }^{1}$ In the following we will use the natural units where $c=h=1$
} 
electromagnetic and hadronic showers, the energy loss in non instrumented material, and the bending of charge particles in the magnetic field. The measured cross sections are unfolded to the particle level and have been compared with pure next to leading order (NLO) predictions, corrected for the nonperturbative effects, and with POWHEG Monte Carlo simulations, which perform a NLO prediction coherently interfaced with parton shower, hadronization and underlying event simulations (ATLAS 2011a).
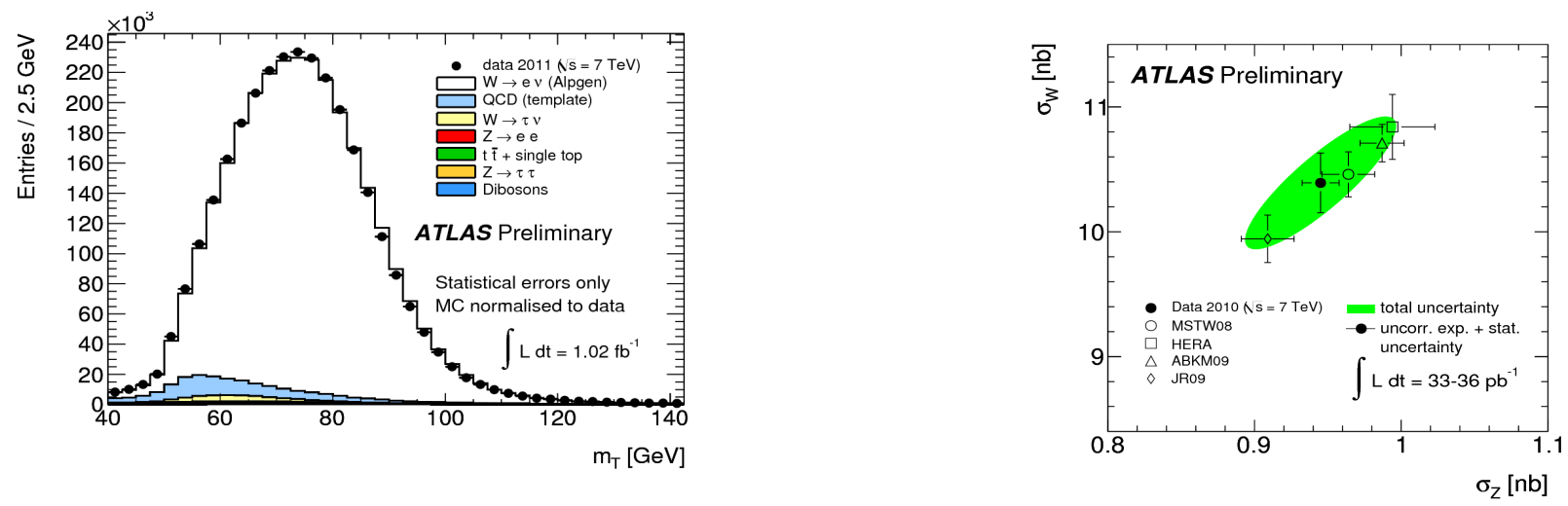

Figure 2: Left: Transverse mass distribution for $W \rightarrow$ ev candidates. QCD background has been determined from data while the other background contributions are derived from Monte Carlo simulations (ATLAS 2011c). Right: $W$ boson measured cross section as a function of the $Z$ boson measured cross section together with the total uncertainty. The theoretical expectations are reported using different Parton Distribution Functions (PDF) (ATLAS 2011c)

In the measurement of the dijet mass cross section (Figure 1: Right), the first two jets, ordered in decreasing $\mathrm{p}_{\mathrm{T}}$, are used to measure the dijet invariant mass $\mathrm{M}_{\mathrm{j}}$, and the angular variable $|\mathrm{y}|_{\max }=$ $\max \left(|\mathrm{y}|_{1} ;|\mathrm{y}|_{2}\right)$. The leading jet $\mathrm{p}_{\mathrm{T}}$ is required to be above $30 \mathrm{GeV}$, and the second above $20 \mathrm{GeV}$. They are both selected in the rapidity range $|y|<2.8$. The dijet mass cross section covers a range from $70 \mathrm{GeV}$ to $4 \mathrm{TeV}$ and shows no evidence of resonance production over background. Limits are set at $95 \% \mathrm{CL}$ for several new physics hypotheses: excited quarks are excluded for masses below $2.99 \mathrm{TeV}$, axigluons are excluded for masses below 3.32 TeV and colour octet scalar resonances are excluded for masses below 1.92 TeV (ATLAS 2011b).

\section{$2.2 W$ and $Z$ boson cross section measurements}

At hadron colliders $W$ and $Z$ bosons can be identified only in their leptonic decay final states. For the $\mathrm{W}$ boson, the presence of the undetected neutrino in the final state gives an event signature with one isolated high $\mathrm{P}_{\mathrm{T}}$ lepton and large missing transverse energy $\left(\mathrm{ME}_{\mathrm{T}}\right)$. The kinematics of the event cannot be completely reconstructed since the longitudinal information of the missing energy is missing. The transverse mass $m_{T}$ is then defined as $m_{T}{ }^{2}=2 E_{T} M E_{T}(2-\cos \varphi)$ where $\varphi$ is the angle between the lepton $p_{T}$ and the missing transverse momentum. The transverse mass is shown (Figure 2: Left) in the $\mathrm{W} \rightarrow$ ev channel with the expected backgrounds. The dominant one is given by "QCD background", (dijet final states) where one jet mimics the electron and the other one is missed or mismeasured giving a large $\mathrm{ME}_{\mathrm{T}}$.

The $Z$ boson final state is characterized by two high $\mathrm{p}_{\mathrm{T}}$, opposite sign and isolated leptons. The $\mathrm{W}$ and $Z$ production cross section have been measured taking into account the background estimations, the trigger and reconstruction efficiencies, the geometric and kinematic acceptance and the integrated luminosity. The results are shown in (Figure 2 Right) together with expectations using different Parton Distribution Functions (PDF) sets.

\section{3 top quark measurements}

The top quark is the heaviest elementary particle of the SM. At the LHC it can be produced in topantitop pairs via the strong interactions or as single top with electroweak processes. In the 
following we will focus on the top quark pair production process. Once produced, the top quark decays with an electroweak process in $t \rightarrow \mathrm{Wb}^{2}$. The different decay modes of the $\mathrm{W}$ boson determine the final state of the top-antitop pair. We can identify three main final states:

- the dilepton one, where both $\mathrm{W}$ decay leptonically in electron or muon. This final state is characterized by an excellent S/B ratio and a small branching fraction (BF)

- the lepton+jet one, where one $\mathrm{W}$ decays in electron/muon and the other hadronically. This final state is characterized by a larger BF and a slightly larger background contamination

- the all-hadronic one, where both $\mathrm{W}$ bosons decay hadronically. This final state is characterized by the largest $\mathrm{BF}$, but the signal is overwhelmed by the QCD multijet production.

ATLAS has measured the top-antitop production cross section in all these decay channels, also including final states with tau lepton. The measured cross section are shown (Figure 4: Left) together with the theoretical expectations (NNLO) for $M_{\text {top }}=172.5 \mathrm{GeV}$ for the dilepton and lepton+jet channel.

The top quark mass is one of the key parameters in the electroweak fit. The three-jet reconstructed top mass is reported in the muon+jet channel with the various background contributions (Figure 4: Right). After combination with the electron channel, the top mass has been measured to be:

$$
M_{\text {top }}=175.9 \pm 0.9(\text { stat. }) \pm 2.7(\text { syst. }) \mathrm{GeV}
$$

So far, in all these measurements measured already with small uncertainties, no deviations from SM expections have been found.
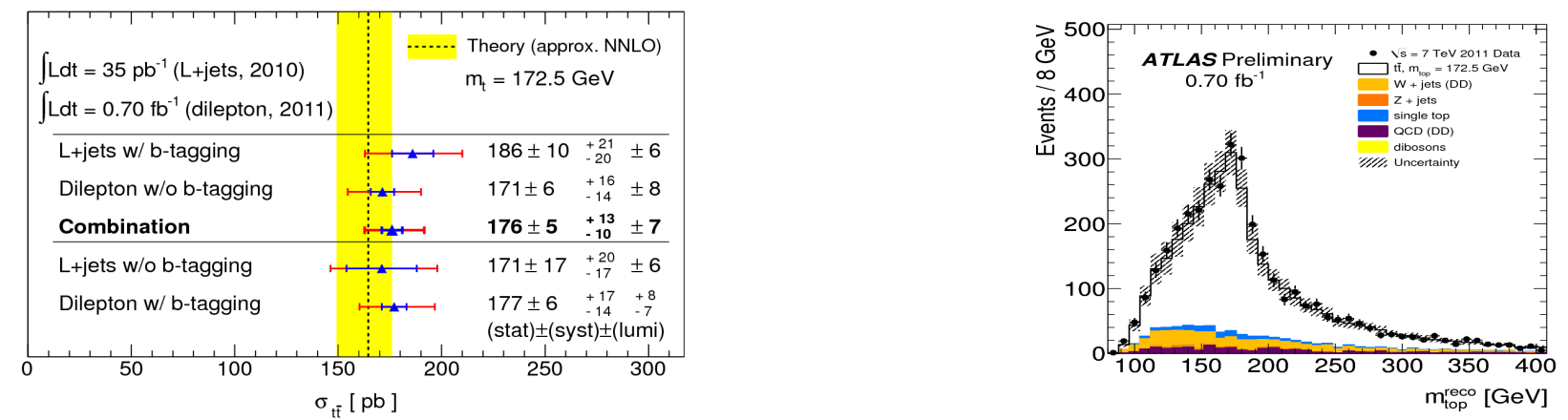

Figure 3: Left: Measured top-anti top cross section for the lepton+jet channel @ $M_{\text {top }}=172.5 \mathrm{GeV}$ with the NNLO theoretical prediction (ATLAS 2011e). Right: Three-jet reconstructed mass in the muon+jet channel. The top quark mass measurement is obtained comparing the data distribution with distributions obtained with different $M_{\text {top }}$ hypothesis (ATLAS 2011f).

\section{Direct Searches}

In this section we will show direct searches of the Standard Model Higgs boson and Supersymmetric processes.

\subsection{SM Higgs boson searches}

Higgs boson discovery or exclusion is one of the main goals of the ATLAS experiment. A review of theoretical predictions can be found in (Djouadi 2011). Looking at the $\sigma \mathrm{XBF}^{3}$ as a function of the Higgs boson mass we can identify two regions: a "high mass region" ( $\left.\mathrm{M}_{\mathrm{H}}>140 \mathrm{GeV}\right)$ where the most sensitive channels are the diboson decays $\mathrm{H} \rightarrow \mathrm{WW}^{(*)}$ or $\mathrm{H} \rightarrow Z Z^{(*)}$ and a "low mass region"

\footnotetext{
${ }^{2}$ Assuming $\left|\mathrm{V}_{\mathrm{tb}}\right|=1$

${ }^{3}$ Production cross section times branching fraction of the decay.
} 
$\left(M_{H}<140 \mathrm{GeV}\right)$ where the most sensitive channels are $\mathrm{H} \rightarrow \mathrm{VY}, \mathrm{H} \rightarrow \mathrm{T} \overline{\mathrm{T}}$ and $\mathrm{H} \rightarrow \mathrm{b} \overline{\mathrm{b}}$ for the Vector Boson Fusion production process with still some contributions from diboson decays.

The most sensitive channel in the "low mass" region is the decay into a pair of photons. This decay channel has a low branching ratio $\left(2 \times 10^{-3}\right.$ for $\left.M_{H}=120 \mathrm{GeV}\right)$, a clear signature and thus a good sensitivity for $M_{H}<140 \mathrm{GeV}$. The search is performed in the $110<\mathrm{M}_{\mathrm{H}}<150 \mathrm{GeV}$ mass range. The candidate events are separated in different categories according to the presence of converted photons and impact points in the calorimeter improving the overall sensitivity. The main backgrounds in this channel are: diphoton production (irreducible), photon-jet production with one fake photon, di-jet production with two fake photons and Drell-Yan events where both electrons are misidentified as photons. The diphoton invariant mass for the $1.08 \mathrm{fb}^{-1}$ data sample is reported (Figure 5: Left) together with five times the expected contribution from a Higgs boson with $M_{H}=120$ $\mathrm{GeV}$. In the "high mass region" one of the most promising channels is the $\mathrm{H} \rightarrow \mathrm{WW}^{(*)}$ decay channel. In particular for the $120<\mathrm{M}_{\mathrm{H}}<200 \mathrm{GeV}$ mass interval the full leptonic channel $\mathrm{H} \rightarrow \mathrm{WW}^{(*)} \rightarrow \mathrm{IVlv}$ is the most sensitive. Given the fact that the Higgs boson is scalar, the angle between the two leptons $\varphi($ II) can be used as a discriminating variable between the SM Higgs boson production process and its main backgrounds (dilepton WW/WZ production, $\mathrm{W}+$ jets, etc.) (Figure 5: Right).
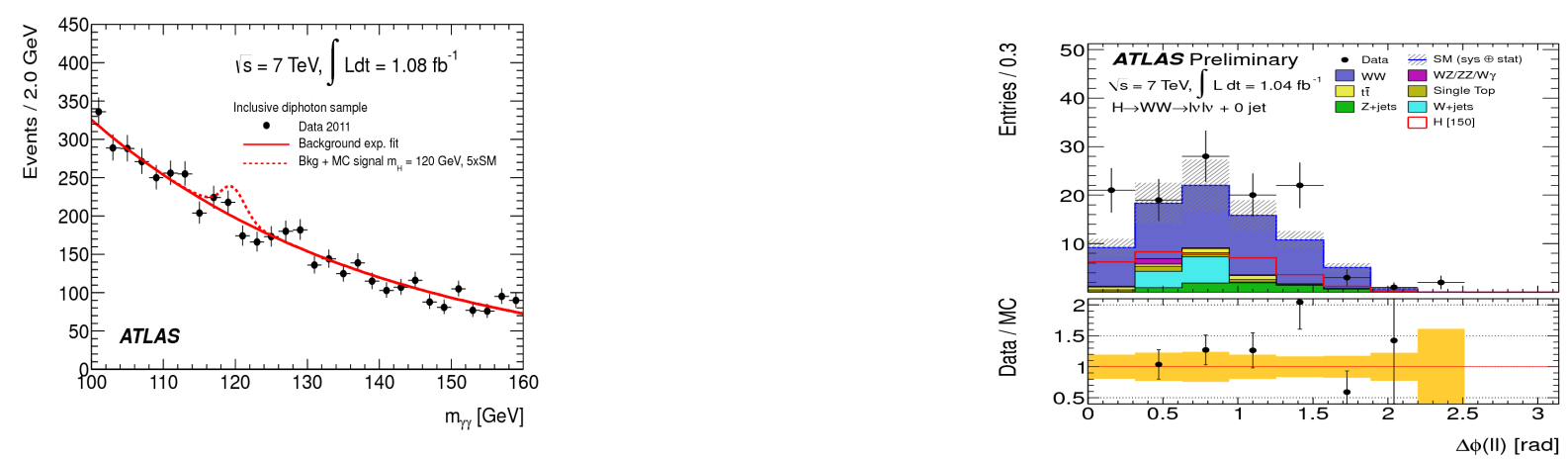

Figure 4: Left: Distribution of the diphoton reconstructed invariant mass. The expected signal for a Higgs boson of $120 \mathrm{GeV}$ mass with five times the SM production cross section is shown (ATLAS 2011g). Right: $H$

$\rightarrow W W^{*)} \rightarrow$ Ivlv selection, 0-jet bin: the azimuthal opening angle $\Delta \varphi / l$ of the two selected leptons after preselection cuts (ATLAS 2011h).

$\mathrm{H} \rightarrow \mathrm{ZZ}^{(*)}$ candidates are searched in the $\mathrm{ZZ} Z^{(*)} \rightarrow \| v v$ and $Z Z^{(*)} \rightarrow \| q q$ final states and the "four lepton golden mode" channel $Z Z^{(*)} \rightarrow \mid l l l$. (Figure 6: Left) shows the four lepton invariant mass with three different Higgs boson mass hypothesis. The former channels contribute with larger BF but with a larger background contamination. (Figure 6: Right) shows the transverse mass distribution for the $\mathrm{H} \rightarrow \mathrm{ZZ}^{(*)} \rightarrow \| \mathrm{lvV}$ candidates.
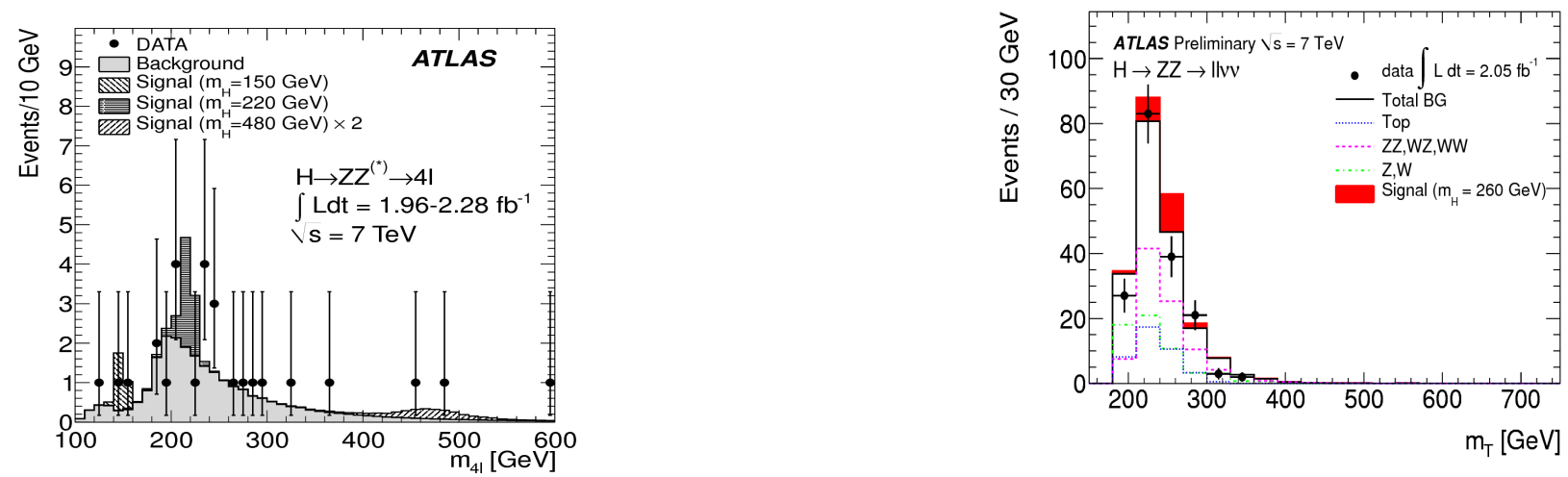

Figure 5: Left: Distribution of the reconstructed invariant mass in the four lepton channel. Invariant mass distributions for different Higgs boson mass hypothesis $\left(M_{H}=150,180\right.$ and $\left.480 \mathrm{GeV}\right)$ are shown (ATLAS 2011i). Right: Transverse mass distribution of the $H \rightarrow Z Z\left(^{*}\right) \rightarrow I l v v$ candidates shown together with the expectations from a Higgs boson mass of $M_{H}=260 \mathrm{GeV}$ (ATLAS 2011j). 
No significant excess of events is found with respect to the expectations from SM processes, therefore exclusion limits on the production cross section are set at 95\% Confidence Level (CL) with a statistical analysis based on the CL method (Read 2011). The contributions from the different channel are shown individually together with their statistical combination (Figure 7: Left). These results have been combined with CMS ones to maximize the overall sensitivity. The observed data are compatible with the background only hypothesis and the SM Higgs boson is excluded at $95 \%$ C.L. or higher in the mass range $141-476 \mathrm{GeV}$. The region from 146 to $443 \mathrm{GeV}$ is excluded at the $99 \%$ C.L (Figure 7: Right).
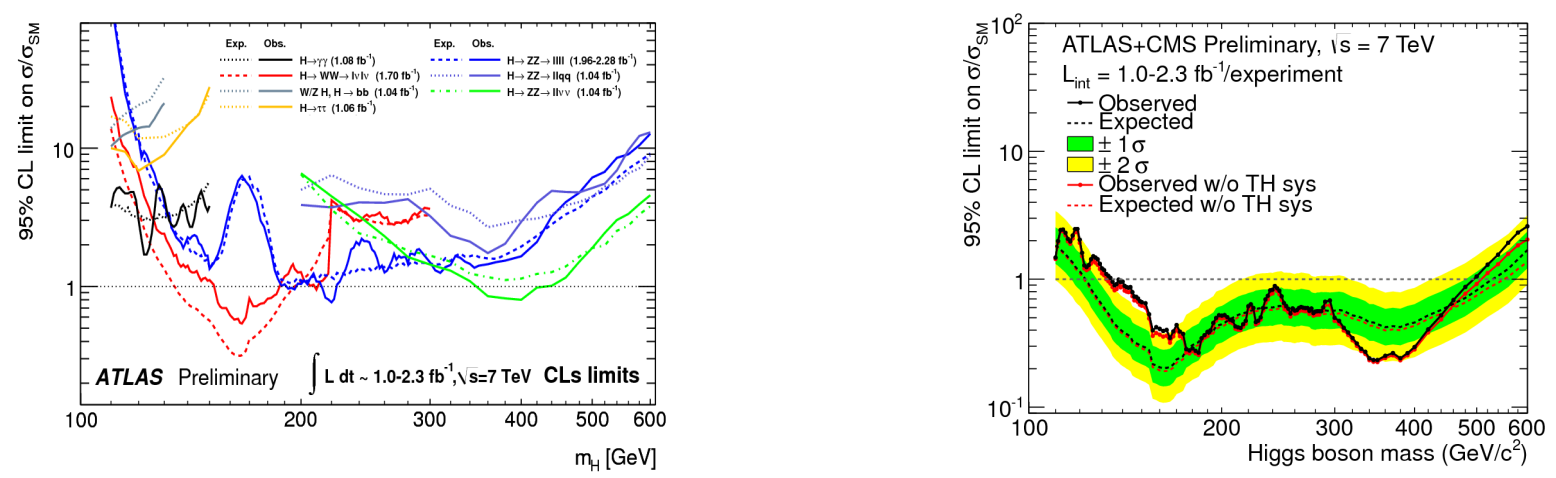

Figure 6: Left The expected (dashed) and observed (solid) cross section 95\% exclusions limits for the individual search channels, normalised to the Standard Model Higgs boson cross section, as functions of the Higgs boson mass (ATLAS 2011k). Right: The combined ATLAS-CMS 95\% C.L. upper limits on the $\sigma / \sigma_{S M}$ ratio obtained with the CLs method, as a function of the SM Higgs boson mass in the range 110-600 GeV. The observed limits are shown by solid symbols. The dashed line indicates the median expected value for the background-only hypothesis, while the green (yellow) bands indicate the ranges expected to contain $68 \%(95 \%)$ of all observed limit excursions from the median. The limits obtained without the theoretical systematic uncertainties are also shown for comparison (ATLAS 2011I).

\subsection{SUSY searches}

SUSY is one of the most popular extensions of the Standard Model of particle physics. Each fundamental Standard Model fermion has a supersymmetric boson partner and vice versa. SUSY solves the mass hierarchy problem. A quantum number $R$ is defined to be $R=(-1)^{2 s+3 B+L}{ }^{4}$. In the following we will focus on the SUSY class of theories where R-parity is conserved. In those cases, the Lightest Supersymmetric Particle (LSP) is produced at the end of all s-particle cascade-decay and is stable. LSP are natural candidates for dark matter. LSP are also weakly interacting and escape detection giving large transverse missing energy. The production of colored $s$-particles could be possible at the LHC giving final states with high $\mathrm{p}_{\mathrm{T}}$ jets. Also additional high $\mathrm{P}_{\mathrm{T}}$ leptons can be produced from model dependent cascades. Final states are then characterized by several high momentum jets, large missing transverse energy and 0, 1 or 2 high momentum leptons. Here we will show the results in the 0-lepton and 1-lepton bin. No excess of events over the background expectation is seen in any signal region, and exclusion limits have been set in the $\left(m_{0}, m_{1 / 2}\right)$ mSUGRA/CMSSM plane (Figure 8). Squarks and gluinos of equal mass have been excluded at $95 \%$ C.L. for masses below $980 \mathrm{GeV}$.

\section{Conclusions}

The ATLAS detector is working well since the start of LHC collisions in 2009. The integrated luminosity of proton-proton collisions delivered by LHC is awesome. We have shown only a minor part of the physics measurement performed by ATLAS. The differential jet cross section as a function of the momentum has been shown together with the dijet mass distribution that excludes several new physics hypotheses. The $W$ and $Z$ cross section has been measured in the electron, muon and tau channel. We have reported the top-antitop cross section measurement in most of the final states and the top mass measurement in the letpon+jet channel. Direct searches of SM

\footnotetext{
${ }^{4}$ With spin S, baryon number B, and lepton number L. All Standard Model particles have Rparity of 1 while supersymmetric particles have R-parity -1
} 
Higgs boson in the most promising final states have been presented. Finally, direct SUSY searches have been shown. The analysis presented there are based at most on $2.28 \mathrm{fb}^{-1}$ and analysis are progressing fast on the remaining data collected in 2011. So far no direct or indirect indications of Higgs boson or Physics Beyond SM have been observed. For 2012 we expect at least to double the statistics collected so far.

\section{Post Scriptum}

At the time of writing of this paper, the ATLAS Collaboration has reported a substantial update on the search for the SM Higgs boson in the gamma-gamma and four-lepton final states, based on the analysis of the full 2011 data, and on their combination with the results already available during the summer. The results of this updated study are shown in (ATLAS 20110)
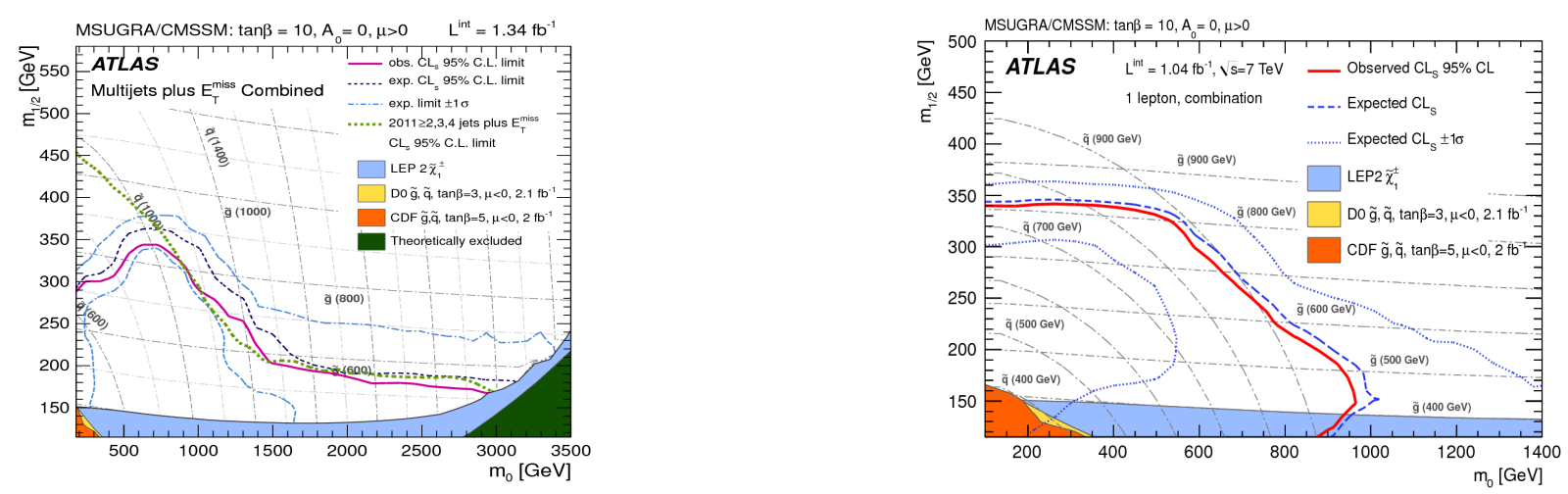

Figure 7: Left: combined exclusion limits from the 0-lepton analysis in the $\left(m_{0}, m_{1 / 2}\right)$ plane for mSUGRA/CMSSM models with $\tan \beta=10, A_{0}=0$ and $\mu>0$. The dashed-blue and the red lines correspond to

the expected and observed 95\% CL limits, respectively. The dotted blue lines correspond to the $\pm 1 \sigma$ variation in the expected limits. The observed ATLAS limit from 2010 analysis $(\tan \beta=3)$ is shown by the solid black line. The star indicates the position of the $m S U G R A$ reference point with $m_{0}=660 \mathrm{GeV}, m_{1 / 2}=240 \mathrm{GeV}$, $A_{0}=0, \tan \beta=10$ and $\mu>0$ (ATLAS 2011m). Right: observed and expected 95\% CL exclusion limits from the 1lepton analysis, as well as the $\pm 1 \sigma$ variation on the expected limit, in the combined electron and muon channels (ATLAS 2011n).

\section{References}

ATLAS (2008) The ATLAS Experiment at the CERN Large Hadron Collider, JINST 3, S08003; ATLAS (2011a), ATL-COM-PHYS-2011-1082, http://cdsweb.cern.ch/record/1375181;

ATLAS (2011b) CERN-PH-EP-2011-127, arXiv:1108.6311 [hep-ex].

ATLAS (2011c) CERN-PH-EP-2011-143, arXiv:1109.5141 [hep-ex];

ATLAS (2011d) CERN-PH-EP-2011-122 arXiv:1108.4101 [hep-ex];

ATLAS (2011e) ATLAS-CONF-2011-108, http://cdsweb.cern.ch/record/1373410; ATLAS (2011f) ATLAS-CONF-2011-120, http://cdsweb.cern.ch/record/1376412;

Djouadi A (2011) Higgs: Theory, in proceedings of Lepton-Photon 2011. Mumbai; Read A. L. (2011), NJ. Phys. G 28, 2963;

ATLAS (2011g) arXiv:1108.5895 [hep-ex];

ATLAS (2011h) ATLAS-CONF-2011-134, http://cdsweb.cern.ch/record/1383837;

ATLAS (2011i) arXiv:1109.5945 [hep-ex];

ATLAS (2011j) arXiv:1109.3357 [hep-ex];

ATLAS (2011k) ATLAS-CONF-2011-135, http://cdsweb.cern.ch/record/1383838;

ATLAS (2011I) ATLAS-CONF-2011-157, http://cdsweb.cern.ch/record/1399599;

ATLAS (2011m) CERN-PH-EP-2011-155, arXiv:1110.2299 [hep-ex];

ATLAS (2011n) CERN-PH-EP-2011-153, arXiv:1109.6606 [hep-ex];

ATLAS (20110) ATLAS-COM-CONF-2011-163,http://cdsweb.cern.ch/record/1406358. 